\title{
Inverse vibration problems for finite-element models
}

\author{
G M L Gladwell $\dagger$ \\ Solid Mechanics Division, Faculty of Engineering, University of Waterloo, Waterloo, Ontario, \\ Canada N2L 3G1
}

Received 14 October 1996

\begin{abstract}
The direct problem of free vibration analysis via finite-element formulation is well known: the element mass and stiffness matrices are assembled to form the system mass and stiffness matrices; the problem is reduced to standard eigenvalue form; the eigenvalues and eigenvectors are found.

The paper discusses some inverse problems relating to finite-element formulations for simple chain-like structures. How can the element mass and stiffness matrices be reconstructed from the overall matrices? How can the overall mass and stiffness matrices be recontructed from the overall dynamic stiffness matrix? How can we find a family of systems having the same eigenvalues?
\end{abstract}

\section{Introduction}

The term inverse vibration problem is used to denote a class of problems in which it is required to construct a vibrating system from specified vibratory behaviour. There are various types of inverse vibration problems, depending on the type of system which is being sought, the kind of vibratory behavioural data which is being matched, and the way in which the problem is viewed, either as a mathematical one with exact, complete data, or as an engineering one with inaccurate incomplete data.

Thus, among inverse vibration problems, there are the well studied and now classical problems, relating to the unique reconstruction of a discrete, undamped in-line system of masses and springs from eigenvalue or frequency response data. Gantmakher and Krein (1950) were the first to study such problems; a review of subsequent research may be found in Gladwell (1986a). Mathematically, this problem reduces to the reconstruction of a symmetric tridiagonal matrix $\mathbf{A}$ from its eigenvalues $\left(\lambda_{i}\right)_{1}^{n}$, and the eigenvalues $\left(\mu_{i}\right)_{1}^{n}$ of its leading principal submatrix $\mathbf{A}_{L}$; Boley and Golub (1987) review the literature.

At the other end of the spectrum of inverse vibration problems are the engineering type of problems usually called finite-element model updating problems, for a review of which see Mottershead and Friswell (1993) or Friswell and Mottershead (1995). The essence of these problems is that there is a finite-element model of a vibrating system, its predictions do not match some experimental behavioural data, and it is required to update the model to improve the match between predictions and experimental data.

Reviews of research on other types of inverse vibration problems may be found in Gladwell (1986b, 1996a), and of other inverse eigenvalue problems in Chu (1995).

This paper is concerned with what could be termed a semi-classical inverse eigenvalue problem. It is classical in the sense that it will be assumed that the system is conservative,

$\dagger$ E-mail address: gladwell@sail.uwaterloo.ca 
and that all its natural frequencies are known exactly. It is not truly classical, in that we do not know what data are sufficient to reconstruct the system exactly. Certainly the natural frequencies and some reference mass alone are insufficient. This means that the emphasis of the paper lies in finding a family, perhaps the complete family, of systems of the given type which match the given eigenvalue data.

The system that we study is the simplest, proper, finite-element model: a finite-element model of an in-line system of elements having one degree of freedom at each end. Such a system has stiffness and mass matrices $\mathbf{K}$ and $\mathbf{M}$ which are symmetric and tridiagonal. The inverse problem consists in constructing the element stiffness and mass matrices, $\mathbf{K}_{\mathrm{e}}$ and $\mathbf{M}_{\mathrm{e}}$, from the natural frequences of the system. The lack of uniqueness in this reconstruction arises on three levels.

(a) Given $\mathbf{K}$ and $\mathbf{M}$, find $\mathbf{K}_{\mathrm{e}}$ and $\mathbf{M}_{\mathrm{e}}$ (section 2).

(b) If $\mathbf{M}=\mathbf{B B}^{\mathrm{T}}$, then the eigenvalue problem $(\mathbf{K}-\lambda \mathbf{M}) \boldsymbol{u}=\mathbf{0}$ may be reduced to $(\tilde{\mathbf{G}}-\lambda \mathbf{I}) \tilde{\boldsymbol{x}}=\mathbf{0}$, where $\tilde{\mathbf{G}}=\mathbf{B}^{-1} \mathbf{K} \mathbf{B}^{-\mathrm{T}}$. The second part of the inverson is therefore: given $\tilde{\mathbf{G}}$, find $\mathbf{K}$ and $\mathbf{M}$ (section 3 ).

(c) The matrix $\tilde{\mathbf{G}}$ is constructed from two tridiagonal matrices $\mathbf{K}$ and $\mathbf{M}$. The third part of the inversion is therefore: given $\tilde{\mathbf{G}}$ constructed from $\mathbf{K}$ and $\mathbf{M}$, find other matrices $\tilde{\mathbf{G}}^{*}$ having the same eigenvalues as $\tilde{\mathbf{G}}$ which may be constructed from other tridiagonal matrices $\mathbf{K}^{*}, \mathbf{M}^{*}$ by $\tilde{\mathbf{G}}^{*}=\mathbf{B}^{*-1} \mathbf{K}^{*} \mathbf{B}^{*-\mathrm{T}}$ (sections 4 and 5 ).

\section{Finite-element models}

Gladwell and Ahmadian (1995) have shown that the generic forms of the element stiffness and mass matrices for an element with two degrees of freedom, one at each end, are

$$
\mathbf{K}_{\mathrm{e}}=\left[\begin{array}{cc}
k_{\mathrm{e}} & -k_{\mathrm{e}} \\
-k_{\mathrm{e}} & k_{\mathrm{e}}
\end{array}\right] \quad \mathbf{M}_{\mathrm{e}}=\left[\begin{array}{cc}
m_{\mathrm{e} 11} & m_{\mathrm{e} 12} \\
m_{\mathrm{e} 21} & m_{\mathrm{e} 22}
\end{array}\right]
$$

where $m_{\mathrm{e} 11}, m_{\mathrm{e} 22}>0, m_{\mathrm{e} 12}=m_{\mathrm{e} 21} \geqslant 0, m_{\mathrm{e} 11} m_{\mathrm{e} 22}-m_{\mathrm{e} 12}^{2}>0$.

First consider the recovery of the element stiffnesses from the overall stiffness matrix. The latter will have the generic form

$$
\mathbf{K}=\left[\begin{array}{cccc}
k_{1}+k_{2} & -k_{2} & & \\
-k_{2} & k_{2}+k_{3} & \ddots & \\
& \ddots & \ddots & k_{n} \\
& & -k_{n} & k_{n}+k_{n+1}
\end{array}\right]
$$

The stiffness $k_{1}\left(k_{n+1}\right)$ will be zero if the left- (right-) hand end is free. We will assume that at least one end of the system is supported, so that $\mathbf{K}$ is positive definite. The matrix $\mathbf{K}$ may be uniquely written as the (finite element type of) superposition of $n+1$ matrices

$$
\left[\begin{array}{ll}
0 & \\
& k_{1}
\end{array}\right] ;\left[\begin{array}{cc}
k_{i} & -k_{i} \\
-k_{i} & k_{i}
\end{array}\right] \quad i=2,3, \ldots, n \quad\left[\begin{array}{cc}
k_{n+1} & 0 \\
0 & 0
\end{array}\right] .
$$

For $\mathbf{M}$, the reconstruction is not unique. Suppose that $\mathbf{M}$ has the form

$$
\mathbf{M}=\left[\begin{array}{cccc}
c_{1} & d_{1} & & \\
d_{1} & c_{2} & \ddots & \\
& \ddots & \ddots & d_{n-1} \\
& & d_{n-1} & c_{n}
\end{array}\right]
$$


where $\mathbf{M}$ is positive definite, and $d_{1}, d_{2}, \ldots, d_{n}>0$. We need to express $\mathbf{M}$ as the superposition of $n$ element mass matrices

$$
\mathbf{M}_{1}=\left[\begin{array}{ll}
0 & 0 \\
0 & f_{1}
\end{array}\right] \quad \mathbf{M}_{2}=\left[\begin{array}{cc}
e_{2} & d_{1} \\
d_{1} & f_{2}
\end{array}\right] \ldots \quad \mathbf{M}_{n}=\left[\begin{array}{cc}
e_{n} & d_{n-1} \\
d_{n-1} & f_{n}
\end{array}\right] .
$$

Thus,

$$
e_{i+1}+f_{i}=c_{i} \quad i=1,2, \ldots, n-1 \quad f_{n}=c_{n} .
$$

The complete solution is a constrained $(n-1)$-parameter family. We choose $f_{1}<c_{1}$, take $e_{2}=c_{2}-f_{1}$, then $f_{2}$ so that $e_{2} f_{2}>d_{1}^{2}, e_{3}=c_{3}-f_{2}$, and so on until $e_{n}$, which must be chosen so that $e_{n} c_{n}>d_{n-1}^{2}$.

We may systematically construct a one-parameter family of solutions by employing the Sturm polynomials for the matrix $\mathbf{M}$. These are

$$
P_{0}(x)=1 \quad P_{1}(c)=c_{1}-x \quad P_{i+1}(x)=\left(c_{i+1}-x\right) P_{i}(x)-d_{i}^{2} P_{i-1}(x)
$$

$i=1,2, \ldots, n-1$. We take $f_{1}=x$, then $e_{2}=c_{1}-x=P_{1}(x)$, and we may choose

$$
\begin{aligned}
& e_{i+1}=P_{i}(x) / P_{i-1}(x) \quad i=1,2, \ldots, n-1 \\
& f_{i+1}=\left(d_{i}^{2} / e_{i+1}\right)+x \quad i=1,2, \ldots, n-2 \quad f_{n}=c_{n} .
\end{aligned}
$$

If $x$ is positive, but not larger than the first eigenvalue of $\mathbf{M}$, then all the $P_{i}(x)$, $i=0,1, \ldots, n-1$, will be positive. Thus all $e_{i}$ and $f_{i}$ will be positive, and

$$
\left|\mathbf{M}_{i+1}\right|=e_{i+1} f_{i+1}-d_{i}^{2}=e_{i+1} x>0 \quad i=1,2, \ldots, n-2
$$

while

$$
\begin{aligned}
\left|\mathbf{M}_{n}\right| & =e_{n} f_{n}-d_{n-1}^{2}=\left(c_{n} P_{n-1}(x)-d_{n-1}^{2} P_{n-2}(x)\right) / P_{n-2}(x) \\
& =\left(P_{n}(x)+x P_{n-1}(x)\right) / P_{n-2}(x)>0 .
\end{aligned}
$$

This answers question (a): given $\mathbf{K}$ and $\mathbf{M}$, find $\mathbf{K}_{\mathrm{e}}$ and $\mathbf{M}_{\mathrm{e}}$.

Gordis (1996) has considered the problem of disassembling $\mathbf{K}$ and $\mathbf{M}$ in a wider context.

\section{Reconstructing $K$ and $M$ from $G$}

The basic eigenvalue problem is

$$
(\mathbf{K}-\lambda \mathbf{M}) \boldsymbol{u}=\mathbf{0} .
$$

We will have to pay considerable attention to the signs of the elements in various matrices. If $\mathbf{P}=\left(p_{i j}\right)$, then we use $\tilde{\mathbf{P}}$ to denote the matrix $\left((-)^{i+j} p_{i j}\right)$. The factorization $\mathbf{M}=\mathbf{B B}^{\mathrm{T}}$ leads to the standard form

$$
(\tilde{\mathbf{G}}-\lambda \mathbf{I}) \tilde{\boldsymbol{x}}=\mathbf{0}
$$

where

$$
\tilde{\mathbf{G}}=\mathbf{B}^{-1} \mathbf{K B}^{-\mathrm{T}} \text { and } \tilde{\boldsymbol{x}}=\mathbf{B}^{\mathrm{T}} \boldsymbol{u} .
$$

(We use $\tilde{\mathbf{G}}$ here because then, as we will show in section 5, $\mathbf{G}$ will have all positive terms.) We now consider the problems of reconstructing $\mathbf{K}$ and $\mathbf{M}$ from $\tilde{\mathbf{G}}$. We can carry out this reconstruction only if $\tilde{\mathbf{G}}$ has the correct form, i.e. it is a product of three matrices $\mathbf{B}^{-1}$, $\mathbf{K}$ and $\mathbf{B}^{-\mathrm{T}}$. For this reason, we change the question. First, however, we define a Jacobi matrix as a symmetric, positive-definite, tridiagonal matrix with positive codiagonal. We can thus state that, for our system, $\tilde{\mathbf{K}}$ and $\mathbf{M}$ are Jacobi matrices. 
We may now rephrase our problem. Given Jacobi matrices $\tilde{\mathbf{K}}$ and $\mathbf{M}$ with

$$
\tilde{\mathbf{K}}=\mathbf{A A}^{\mathrm{T}} \quad \mathbf{M}=\mathbf{B B}^{\mathrm{T}}
$$

where $\mathbf{A}$ and $\mathbf{B}$ are lower bidiagonal matrices with positive diagonals and codiagonals, find Jacobi matrices $\tilde{\mathbf{K}}^{*}$ and $\mathbf{M}^{*}$ with

$$
\tilde{\mathbf{K}}^{*}=\mathbf{C C}^{\mathrm{T}} \quad \mathbf{M}^{*}=\mathbf{D D}^{\mathrm{T}}
$$

such that $\mathbf{C}$ and $\mathbf{D}$ are lower bidiagonal matrices with positive diagonals and codiagonals, and

$$
\tilde{\mathbf{G}}=\mathbf{B}^{-1} \mathbf{K} \mathbf{B}^{-\mathrm{T}}=\mathbf{D}^{-1} \mathbf{K}^{*} \mathbf{D}^{-\mathrm{T}} .
$$

Equation (3.2) is equivalent to

$$
\mathbf{G}=\tilde{\mathbf{B}}^{-1} \mathbf{A} \mathbf{A}^{\mathrm{T}} \tilde{\mathbf{B}}^{-\mathrm{T}}=\tilde{\mathbf{D}}^{-1} \mathbf{C} \mathbf{C}^{\mathrm{T}} \mathbf{D}^{-\mathrm{T}} .
$$

The positive definite matrix $\mathbf{G}$ has a unique Cholesky factorization $\mathbf{G}=\mathbf{L L}^{\mathrm{T}}$, with positive diagonal. Thus (3.3) holds if

$$
\tilde{\mathbf{B}}^{-1} \mathbf{A}=\mathbf{L}=\tilde{\mathbf{D}}^{-1} \mathbf{C} \text {. }
$$

Straightforward algebra shows that this implies

$$
\begin{aligned}
& c_{i i}=v_{i} a_{i i} \quad d_{i i}=v_{i} b_{i i} \quad i=1,2, \ldots, n \\
& c_{i+1, i}=v_{i+1} a_{i+1, i} \quad d_{i+1, i}=v_{i+1} b_{i+1, i} \quad i=2,3, \ldots, n-1
\end{aligned}
$$

and

$$
\frac{d_{21} c_{11}}{d_{11} d_{22}}+\frac{c_{21}}{d_{22}}=\frac{b_{21} a_{11}}{b_{11} b_{22}}+\frac{a_{21}}{b_{22}}
$$

Equation (3.6) may be reduced to

$$
a_{11} d_{21}+b_{11} c_{21}=v_{2} f_{1}
$$

where

$$
f_{1}=a_{11} b_{21}+a_{21} b_{11} .
$$

This has the general positive solution

$$
c_{21}=v_{2} f_{1} \sin ^{2} \theta / b_{11} \quad d_{21}=v_{2} f_{1} \cos ^{2} \theta / a_{11}
$$

where $0<\theta<\pi / 2$.

This provides an $(n+1)$-parameter family of Jacobi matrices $\tilde{\mathbf{K}}^{*}$ and $\mathbf{M}^{*}$ specified by the $n$ positive parameters $v_{i}$ and the angle $\theta$.

Unless the parameters $v_{i}$ are chosen properly, $\mathbf{K}^{*}$ will not have the generic form (2.1) of a stiffness matrix. Matrices of this form are characterized by the equation

$$
\mathbf{K}\{1,1, \ldots, 1\}=\left\{k_{1}, 0, \ldots, 0, k_{n+1}\right\} .
$$

This states that forces $k_{1}$ and $k_{n+1}$ applied to the two ends of the system will displace all the joints to the right by one unit.

The solution (3.4)-(3.7) gives

$$
\mathbf{C}=\gamma \mathbf{C}_{0}
$$

where $\mathbf{C}_{0}$ is determined once $\theta$ has been chosen, and $\gamma=\operatorname{diag}\left\{\gamma_{1}, \gamma_{2}, \ldots, \gamma_{n}\right\}$. If $\mathbf{K}^{*}$ is to be a proper stiffness matrix, then

$$
\mathbf{K}^{*}\{1,1, \ldots, 1\}=\left\{k_{1}^{*}, \ldots, k_{n+1}^{*}\right\} .
$$


Since $\mathbf{K}^{*}=\tilde{\mathbf{C}} \tilde{\mathbf{C}}^{\mathrm{T}}=\gamma \tilde{\mathbf{C}}_{0} \tilde{\mathbf{C}}_{0}^{\mathrm{T}} \gamma$, we may write (3.7) as

$$
\gamma \tilde{\mathbf{C}}_{0} \tilde{\mathbf{C}}_{0} \gamma\{1,1, \ldots, 1\}=\left\{k_{1}^{*}, \ldots, k_{n+1}^{*}\right\}
$$

i.e.

$$
\left\{\gamma_{1}, \gamma_{2}, \ldots, \gamma_{n}\right\}=\tilde{\mathbf{C}}_{0}^{-1} \tilde{\mathbf{C}}_{0}^{-\mathrm{T}}\left\{k_{1}^{*} / \gamma_{1}, 0, \ldots, 0, k_{n+1}^{*} / \gamma_{n}\right\}
$$

The matrix $\tilde{\mathbf{C}}_{0}^{-1}$ is a lower triangular matrix with all positive terms, so that $\mathbf{K}_{0}^{-1}=$ $\tilde{\mathbf{C}}_{0}^{-1} \tilde{\mathbf{C}}_{0}^{-\mathrm{T}}$ is a symmetric matrix with all positive terms. We write $\mathbf{K}_{0}^{-1}=\mathbf{P}=$ $\left\{\boldsymbol{p}_{1}, \boldsymbol{p}_{2}, \ldots, \boldsymbol{p}_{n}\right\}$. Then the solution of (3.9) is

$$
\left\{\gamma_{1}, \gamma_{2}, \ldots, \gamma_{n}\right\}=\left(k_{1}^{*} / \gamma_{1}\right) \boldsymbol{p}_{1}+\left(k_{n+1}^{*} / \gamma_{n}\right) \boldsymbol{p}_{n} .
$$

This gives the family of possible solutions. First we find $\gamma_{1}$ and $\gamma_{n}$, then the remainder $\gamma_{2}, \ldots, \gamma_{n-1}$, from (3.9). We have

$$
\gamma_{1}=\frac{k_{1}^{*}}{\gamma_{1}} p_{11}+\frac{k_{n+1}^{*}}{\gamma_{n}} p_{1 n} \quad \gamma_{n}=\frac{k_{1}^{*}}{\gamma_{1}} p_{n 1}+\frac{k_{n+1}^{*}}{\gamma_{n}} p_{n n} .
$$

We may take $\gamma_{1}=1$, and choose $k_{1}^{*}$ arbitrarily provided that $0<k_{1}^{*}<1 / p_{11}$, then

$$
\gamma_{n}=\frac{p_{n n}\left(1-p_{11} k_{1}^{*}\right)+p_{n 1} p_{1 n} k_{1}^{*}}{p_{n 1}} \quad k_{n+1}^{*}=\gamma_{n} \frac{1-k_{1}^{*} p_{11}}{p_{1 n}} .
$$

We have completed the reconstruction of $\mathbf{K}$ and $\mathbf{M}$ from $\mathbf{G}$.

\section{A simple isospectral set of systems}

Equation (3.1) and (3.2) show that the basic eigenvalue problem is

$$
\left(\tilde{\mathbf{B}}^{-1} \mathbf{A} \cdot \mathbf{A}^{-\mathrm{T}} \tilde{\mathbf{B}}^{-\mathrm{T}}-\lambda \mathbf{I}\right) \boldsymbol{x}=\mathbf{0} .
$$

It is a standard result of matrix algebra that if $\mathbf{G}$ and $\mathbf{H}$ are square matrices of order $n$, then $\mathbf{G H}$ and $\mathbf{H G}$ have the same eigenvalues, apart perhaps from zero. Since equation (4.1) has non-zero eigenvalues, we can create an isospectral system (one with the same eigenvalues) by reversing the order of the factors in (4.1) to give the system

$$
\left(\mathbf{A}^{\mathrm{T}} \tilde{\mathbf{B}}^{-\mathrm{T}} \cdot \tilde{\mathbf{B}}^{-1} \mathbf{A}-\lambda \mathbf{I}\right) \boldsymbol{y}=\mathbf{0} \quad \boldsymbol{y}=\left(\tilde{\mathbf{B}}^{-1} \mathbf{A}\right)^{-1} \tilde{\boldsymbol{x}} .
$$

This new matrix does not have the same form as $\mathbf{G}$; now the bidiagonal factors are on the outside, and the reciprocals are inside. We therefore seek lower bidiagonal matrices $\mathbf{C}$ and D with positive terms, such that

$$
\tilde{\mathbf{B}}^{-1} \mathbf{A}=\mathbf{C} \tilde{\mathbf{D}}^{-1}
$$

i.e. such that

$$
\mathbf{A D}=\tilde{\mathbf{B} C} .
$$

If we can find such $\mathbf{C}$ and $\mathbf{D}$, then we can write (4.2) as

$$
\left(\tilde{\mathbf{D}}^{-\mathrm{T}} \mathbf{C}^{\mathrm{T}} \mathbf{C} \tilde{\mathbf{D}}^{-1}-\lambda \mathbf{I}\right) \boldsymbol{y}=\mathbf{0}
$$

which we may rewrite as

$$
\left(\tilde{\mathbf{C}} \tilde{\mathbf{C}}-\mathbf{D}^{\mathrm{T}} \mathbf{D}\right) \boldsymbol{v}=\mathbf{0} \quad \boldsymbol{v}=\mathbf{D}^{-1} \tilde{\boldsymbol{y}}=\tilde{\mathbf{C}}^{-1} \boldsymbol{x}=\tilde{\mathbf{C}}^{-1} \mathbf{B}^{\mathrm{T}} \boldsymbol{u} .
$$

Thus we may put

$$
\mathbf{K}^{*}=\tilde{\mathbf{C}}^{\mathrm{T}} \tilde{\mathbf{C}} \quad \mathbf{M}^{*}=\mathbf{D}^{\mathrm{T}} \mathbf{D} .
$$


The matrices $\tilde{\mathbf{K}}^{*}$ and $\mathbf{M}^{*}$ will be Jacobi matrices.

We now examine equation (4.3). Each side is the product of lower bidiagonal matrices; that on the left is

$$
\left[\begin{array}{ccccc}
a_{11} & & & & \\
a_{21} & a_{22} & & & \\
& a_{32} & a_{33} & & \\
& & \ddots & \ddots & \\
& & & a_{n, n-1} & a_{n n}
\end{array}\right]\left[\begin{array}{ccccc}
d_{11} & & & & \\
-d_{21} & d_{22} & & & \\
& -d_{32} & d_{33} & & \\
& & \ddots & \ddots & \\
& & & -d_{n, n-1} & d_{n n}
\end{array}\right] .
$$

Equation (4.3) thus yields $n$ separate sets of equations; the $i$ th set involves the elements in the $i$ th columns of $\mathbf{D}$ and $\mathbf{C}$. For $i=1,2, \ldots, n-2$, the $i$ th set is

$$
\begin{aligned}
& a_{i i} d_{i i}=b_{i i} c_{i i} \quad a_{i+2, i+1} d_{i+1, i}=b_{i+2, i+1} c_{i+1, i} \\
& a_{i+1, i} d_{i i}-a_{i+1, i+1} d_{i+1, i}=-b_{i+1, i} c_{i i}+b_{i+1, i+1} c_{i+1, i} .
\end{aligned}
$$

For $i=n-1$, the second of these equations is missing; for $i=n$, there is just the first equation.

To solve these equations, we note that for $i \leqslant n-2$, we can take one element in the $i$ th pair of columns of $\mathbf{C}$ and $\mathbf{D}$ arbitrarily, and determine the remaining three elements. We write

$$
c_{i i}=\gamma_{i} a_{i i} \quad d_{i i}=\gamma_{i} b_{i i} \quad \gamma_{i}>0 \quad i=1,2, \ldots, n
$$

and find

$c_{i+1, i}=\gamma_{i} \tau_{i} a_{i+2, i+1} \quad d_{i+1, i}=\gamma_{i} \tau_{i} b_{i+2, i+1} \quad i=1,2, \ldots, n-2$

where

$$
\tau_{i}=f_{i} / f_{i+1} \quad f_{i}=a_{i i} b_{i+1, i}+a_{i+1, i} b_{i i} .
$$

This is the general solution for $i \leqslant n-2$.

For $i=n-1$, we use (4.7) in (4.6) to give

$$
a_{n n} d_{n, n-1}+b_{n n} c_{n, n-1}=\gamma_{n-1} f_{n-1}
$$

for which the general positive solution is

$$
c_{n, n-1}=\gamma_{n-1} f_{n-1} \sin ^{2} \theta / b_{n n} \quad d_{n, n-1}=\gamma_{n-1} f_{n-1} \cos ^{2} \theta / a_{n n} .
$$

This analysis provides an $(n+1)$-parameter family of pairs of Jacobi matrices $\tilde{\mathbf{K}}^{*}$ and $\mathbf{M}^{*}$, such that the system $\mathbf{K}^{*}$ and $\mathbf{M}^{*}$ is isospectral to $\mathbf{K}$ and $\mathbf{M}$. The solution (4.7)-(4.10) gives

$$
\tilde{\mathbf{C}}=\tilde{\mathbf{C}}_{0} \gamma
$$

where $\mathbf{C}_{0}$ depends only on $\theta$, and $\gamma=\operatorname{diag}\left(\gamma_{1}, \ldots, \gamma_{n}\right)$. Again, as in section $3, \gamma$ may be chosen to make $\mathbf{K}^{*}=\tilde{\mathbf{C}}^{\mathrm{T}} \tilde{\mathbf{C}}=\gamma^{\mathrm{T}} \tilde{\mathbf{C}}_{0}^{\mathrm{T}} \tilde{\mathbf{C}}_{0} \gamma$ have the generic form (2.1).

\section{A wider isospectral family of systems}

To obtain a larger family of systems which are isospectral to $\mathbf{K}$ and $\mathbf{M}$, we use some recent results on the preservation of signs in a matrix under shifted $\mathbf{Q R}$ factorization and reversal. In this section we state, without proof, some results obtained in Gladwell (1996b). First, however, we introduce some terminology.

If $1 \leqslant k \leqslant n, Q_{k, n}$ will denote the set of strictly increasing sequences $\alpha=$ $\left\{\alpha_{1}, \alpha_{2}, \ldots, \alpha_{k}\right\}$ taken from $\{1,2, \ldots, n\}$. 
Let $\mathbf{A} \in \mathcal{R}^{n \times n}$ and $\alpha, \beta \in Q_{k, n}$. The minor of $\mathbf{A}$ taken from rows $\alpha$ and columns $\beta$ is denoted by $A(\alpha ; \beta)$. Following Karlin (1968) we say that $\mathbf{A}$ is

(i) TP (totally positive) if all minors of $\mathbf{A}$ are non-negative;

(ii) NTP if $\mathbf{A}$ is non-singular and TP;

(iii) STP (strictly TP) if all minors are strictly positive;

(iv) $\mathrm{O}$ (oscillatory) if $\mathbf{A}$ is TP, and $\mathbf{A}^{m}$ is STP for some positive integer $m$. It is known that $\mathbf{A}$ is $\mathrm{O}$ iff $\mathbf{A}$ is NTP and $a_{i, i+1}>0, a_{i+1, i}>0$ for $i=1,2, \ldots, n-1$.

Note that the phrase all minors means all the minors of order 1, the elements of the matrix, and all those of order $2,3, \ldots, n$ are non-negative or positive, as the case may be. These conditions are stricter than those for a positive definite (PD) matrix: $\mathbf{A}$ is PD if the principal minors $A(\alpha ; \alpha)$ are positive. Clearly a symmetric STP matrix is PD; it can be shown that a symmetric TP matrix is PD, but the converse of this statement is false.

We will sometimes use SY to denote symmetric.

Consider some examples. A Jacobi matrix, as we have defined it, is O. It is NTP because any non-zero minor is the product of principal minors, which are positive, and/or codiagonal terms, which are positive. It is $\mathrm{O}$ because it is NTP and because $a_{i, i+1}, a_{i+1, i}>0$.

One of the basic results about oscillatory matrices is that $\mathbf{A}$ is $\mathrm{O}$ iff $\tilde{\mathbf{A}}^{-1}$ is $\mathrm{O}$. This is a direct consequence of the formula for the elements of the adjoint $\hat{\mathbf{A}}$, as $(-)^{i+j} *$ (minor of $\mathbf{A}$ ). This means that $\mathbf{A}^{-1}$ is $\mathrm{O}$ iff $\tilde{\mathbf{A}}$ is $\mathrm{O}$. In particular, therefore, the reciprocal, $\mathbf{K}^{-1}$, of the stiffness matrix in (2.1) is $\mathrm{O}$, because $\tilde{\mathbf{K}}$ is a Jacobi matrix.

The product of two NTP matrices is NTP, and the product of two $\mathrm{O}$ matrices is $\mathrm{O}$. Therefore, if $\tilde{\mathbf{K}}$ and $\mathbf{M}$ are Jacobi matrices, then all the matrices $\mathbf{K}^{-1} \mathbf{M}, \mathbf{M} \mathbf{K}^{-1}, \tilde{\mathbf{M}}{ }^{-1} \tilde{\mathbf{K}}$, and $\tilde{\mathbf{K}} \tilde{\mathbf{M}}^{-1}$ are $\mathrm{O}$. Let us show that the matrix $\mathbf{G}=\tilde{\mathbf{B}}^{-1} \tilde{\mathbf{K}} \tilde{\mathbf{B}}^{-\mathrm{T}}$ is $\mathrm{O}$. The lower bidiagonal matrix $\mathbf{B}$ is obtained by factorizing $\mathbf{M}=\mathbf{B B}^{\mathrm{T}}$. Since $\mathbf{M}$ is $\mathbf{O}, \mathbf{B}$ has positive diagonal and codiagonal, and so is NTP. Therefore, $\tilde{\mathbf{B}}^{-1}$ is NTP, and so is the product of the NTP matrices $\tilde{\mathbf{B}}^{-1}, \tilde{\mathbf{K}}$ and $\tilde{\mathbf{B}}^{-\mathrm{T}}$. Thus $\mathbf{G}$ is NTP. To prove that it is $\mathrm{O}$, we must show that $g_{i, i+1}\left(=g_{i+1, i}\right)>0$, for $i=1,2, \ldots, n-1$. Temporarily put $\tilde{\mathbf{B}}^{-1}=\mathbf{C}, \tilde{\mathbf{K}}=\mathbf{D}$ then using Einstein's summation convention we may write

$$
g_{i, i+1}=c_{i j} d_{j k} c_{i+1, k} .
$$

Every element in this product is non-negative, and in particular the elements $c_{i i}, d_{i, i+1}$, and $c_{i+1, i+1}$ are strictly positive. Thus

$$
g_{i, i+1} \geqslant c_{i i} d_{i, i+1} c_{i+1, i+1}>0
$$

and $\mathbf{G}$ satisfies the criterion for $\mathrm{O}$. Actually, $\mathbf{G}$ is also a positive matrix; all its elements are strictly positive. This follows because $\mathbf{C}\left(=\tilde{\mathbf{B}}^{-1}\right)$ is a strictly positive lower triangle and $\mathbf{C}^{\mathrm{T}}$ is a strictly positive upper triangle; $g_{i j}=c_{i k} d_{k l} c_{j l}$ so that if $i \geqslant j$ then $g_{i j} \geqslant c_{i j} d_{j j} c_{j j}>0$, while if $i \leqslant j, g_{i j} \geqslant c_{i i} d_{i i} c_{j i}>0$.

The theory regarding oscillatory matrices is based on two theorems: Perron's theorem, that a positive matrix $\left(a_{i j}>0\right)$ has at least one real positive eigenvalue, which is larger than the modulus of any other (possibly complex) eigenvalue; and the Binet Cauchy theorem which states that if $\mathbf{A}=\mathbf{B C}$, and $\mathbf{A}_{p}, \mathbf{B}_{p}, \mathbf{C}_{p}$ are the $p$ th compound matrices of $\mathbf{A}, \mathbf{B}$, and $\mathbf{C}$, respectively, then $\mathbf{A}_{p}=\mathbf{B}_{p} \mathbf{C}_{p}$. Remember that if $\mathbf{A}$ is $n \times n$, then $\mathbf{A}_{p}$ is the matrix of order $N \times N$, where $N=\left(\begin{array}{l}n \\ p\end{array}\right)$, constructed from the $p$ th-order minors of $\mathbf{A}$, arranged in lexical order.

A real square symmetric matrix has real eigenvalues. The theorems of Perron and Binet Cauchy yield the fundamental result, that eigenvalues of an oscillatory matrix (whether symmetric or not) are real, distinct and positive. Thus when, as is so in our case, $\tilde{\mathbf{K}}$ and $\mathbf{M}$ 
are Jacobi matrices, the eigenvalues of (3.1) satisfy

$$
0<\lambda_{1}<\lambda_{2}<\cdots<\lambda_{n} .
$$

This is a fundamental result: the finite-element model of an inline system given by $\mathbf{K}$ in (2.1) and $\mathbf{M}$ in (2.2) always has distinct eigenvalues.

We now come to the result proved in Gladwell (1996b). If $\mathbf{A} \in \mathcal{R}^{n \times n}$ and $\mu$ is not an eigenvalue of $\mathbf{A}$, there is a unique factorization

$$
\mathbf{A}-\mu \mathbf{I}=\mathbf{Q R}
$$

where $\mathbf{Q}$ is orthogonal and $\mathbf{R}$ is upper triangular with positive diagonal. We can therefore define an operator $\mathcal{G}_{\mu}: \mathbf{A} \rightarrow \mathbf{A}^{\prime}=\mathbf{A}^{\prime}(\mu)$ where

$$
\mathbf{A}^{\prime}-\mu \mathbf{I}=\mathbf{R} \mathbf{Q} \text {. }
$$

The matrix $\mathbf{A}^{\prime}$ is a unique function of $\mathbf{A}$ and $\mu$. Equations (5.1) and (5.2) have two simple consequences:

$$
\begin{aligned}
& \mathbf{A}^{\prime}=\mu \mathbf{I}+\mathbf{R} \mathbf{Q}=\mu \mathbf{I}+\mathbf{Q}^{\mathrm{T}} \mathbf{Q}(\mathbf{R} \mathbf{Q})=\mu \mathbf{I}+\mathbf{Q}^{\mathrm{T}}(\mathbf{Q R}) \mathbf{Q} \\
& \quad=\mu \mathbf{I}+\mathbf{Q}^{\mathrm{T}}(\mathbf{A}-\mu \mathbf{l}) \mathbf{Q}=\mathbf{Q}^{\mathrm{T}} \mathbf{A} \mathbf{Q} \\
& \mathbf{A}^{\prime} \mathbf{R}=\mu \mathbf{R}+(\mathbf{R} \mathbf{Q}) \mathbf{R}=\mu \mathbf{R}+\mathbf{R}(\mathbf{Q R})=\mathbf{R}(\mu \mathbf{I}+(\mathbf{Q R}))=\mathbf{R A} .
\end{aligned}
$$

Equation (5.3) shows that $\mathbf{A}$ and $\mathbf{A}^{\prime}$ are unitarily equivalent; they have the same eigenvalues. It also shows that $\mathbf{A}^{\prime}$ is symmetric iff $\mathbf{A}$ is symmetric, and that $\mathbf{A}^{\prime}$ is positive definite iff $\mathbf{A}$ is positive definite.

Equation (5.4) shows that if $\mathbf{A}$ is symmetric and banded, than $\mathbf{A}^{\prime}$ has the same bandwidth as $\mathbf{A}$. In particular, if $\mathbf{A}$ is symmetric tridiagonal, then so is $\mathbf{A}^{\prime}$.

The theorem proved in Gladwell (1996b) refers to a symmetric matrix $\mathbf{A}$ with one of the three properties NTP, O, and STP; we label these generically as P. We have proved:

Theorem 1. $\quad \mathbf{A}^{\prime}$ has property SYP iff $\mathbf{A}$ has the corresponding property SYP.

In particular therefore, if $\mathbf{A}$ is symmetric and oscillatory (SYO) and $\mu$ is not an eigenvalue of $\mathbf{A}$, then $\mathbf{A}^{\prime}$ is symmetric and oscillatory. A special case of this is that if $\mathbf{A}$ is a Jacobi matrix, and $\mu$ is not an eigenvalues of $\mathbf{A}$, then $\mathbf{A}^{\prime}$ is a Jacobi matrix.

We now examine the consequences of equation (5.4). Since $\mathbf{R}$ is upper triangular, equation (5.4) yields

$$
\sum_{k=1}^{j} g_{i k}^{\prime} r_{k j}=\sum_{k=i}^{n} r_{i k} g_{k j}
$$

In particular, if $i=n, j=1$, then

$$
g_{n 1}^{\prime} r_{11}=r_{n n} g_{n 1} \text {. }
$$

Since the diagonal of $\mathbf{R}$ is positive, we deduce that $g_{n 1}^{\prime}>0(=0)$ iff $g_{n 1}>0(=0)$. Taking $j=1$ in (3.5) we have

$$
g_{i 1}^{\prime} r_{11}=\sum_{k=i}^{n} r_{i k} g_{k 1}
$$

from which we deduce that if $g_{i 1}, g_{i+1,1}, \ldots, g_{n 1}$ are all zero, then so are $g_{i 1}^{\prime}, g_{i+1,1}^{\prime}, \ldots, g_{n 1}^{\prime}$. Taking $j=2$ in (5.5) we have

$$
g_{i 1}^{\prime} r_{12}+g_{i 2}^{\prime} r_{22}=\sum_{k=i}^{n} r_{i k} g_{k 2}
$$


from which we deduce that if $g_{i 1}^{\prime}, g_{i+1,1}^{\prime}, \ldots, g_{n 1}^{\prime}$ are zero and $g_{i 2}, g_{i+1,2}, \ldots, g_{n 2}$ are zero, then so are $g_{i 2}^{\prime}, g_{i+1,2}^{\prime}, \ldots, g_{n 2}^{\prime}$. These results concern the first two columns of $\mathbf{A}^{\prime}$. We can clearly generalize them to the third and subsequent columns. When $\mathbf{A}$ is symmetric, the results concerning the columns in the lower triangles of $\mathbf{A}$ and $\mathbf{A}^{\prime}$ will yield analogous results for the rows in the upper triangles. We see that if the columns of $\mathbf{A}$ in the lower triangles have a non-increasing pattern of zeros, as in figure 1, then $\mathbf{A}^{\prime}$ has the same pattern.

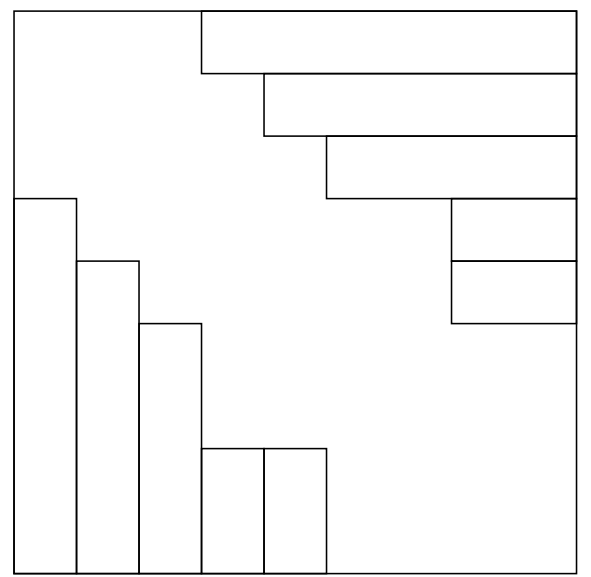

Figure 1. The zero elements of $\mathbf{A}$ are contained in the rectangles on the lower left and the upper right.

The preservation of these patterns of zeros was deduced from equation (5.4). We can deduce analogous results concerning the preservation of pattern of zeros of the minors of $\mathbf{A}$ and $\mathbf{A}^{\prime}$ by using the equation

$$
\mathbf{A}_{p}^{\prime} \mathbf{R}_{p}=\mathbf{R}_{p} \mathbf{A}_{p}
$$

which follows from applying the Binet Cauchy theorem to (5.4). Since $\mathbf{R}_{p}$ is an upper triangular matrix (of order $N$ ) with positive diagonal, equation (5.7) shows that $\mathcal{G}_{\mu}$ will preserve any non-increasing pattern of zeros of the columns in the lower triangle of $\mathbf{A}_{p}$, and if $\mathbf{A}$ is symmetric, the corresponding pattern of zeros of the rows in the upper triangle.

In the analysis we have just described, we have used the symbol $\mathbf{A}$ to denote a symmetric matrix of order $n$. Now we will apply these results to the symmetric matrix $\mathbf{G}$, which we have shown is oscillatory $(\mathrm{O})$. We revert to our former use of $\mathbf{A}$ as a lower bidiagonal matrix with positive diagonal and codiagonal. We will show that if the oscillatory matrix $\mathbf{G}$ is obtained as in (3.3), i.e.

$$
\mathbf{G}=\tilde{\mathbf{B}}^{-1} \mathbf{A A}^{\mathrm{T}} \tilde{\mathbf{B}}^{-\mathrm{T}}
$$

from the two Jacobi matrices $\tilde{\mathbf{K}}=\mathbf{A A}^{\mathrm{T}}$ and $\mathbf{M}=\mathbf{B B}^{\mathrm{T}}$, and if $\mu$ is not an eigenvalue of $\mathbf{G}$, then $\mathbf{G}^{\prime}(\mu)$, which is oscillatory by theorem 1 , can also be factorized in the form

$$
\mathbf{G}^{\prime}=\tilde{\mathbf{B}}^{\prime-1} \mathbf{A}^{\prime} \mathbf{A}^{\prime \mathrm{T}} \tilde{\mathbf{B}}^{\prime-\mathrm{T}}
$$

where $\tilde{\mathbf{K}}^{\prime}=\mathbf{A}^{\prime} \mathbf{A}^{\prime \mathrm{T}}$ and $\mathbf{M}^{\prime}=\mathbf{B}^{\prime} \mathbf{B}^{\prime T}$ are Jacobi matrices.

We need to establish two results: that $\mathbf{G}^{\prime}$ can be factorized in such a way that $\mathbf{A}^{\prime}$ and $\mathbf{B}^{\prime}$ are lower bidiagonal; and that $\mathbf{A}^{\prime}$ and $\mathbf{B}^{\prime}$ both have positive diagonal and codiagonal. We consider the results in that order. 
To establish the band forms, we consider how $\mathbf{G}$ was constructed: $\mathbf{G}=\tilde{\mathbf{B}}^{-1} \tilde{\mathbf{K}} \tilde{\mathbf{B}}^{-\mathrm{T}}$. We write this $\tilde{\mathbf{G}}=\mathbf{B}^{-1} \mathbf{K B}^{-\mathrm{T}}$ or $\mathbf{K}=\mathbf{B} \tilde{\mathbf{G}} \mathbf{B}^{\mathrm{T}}$. This we can write as $\mathbf{C}=\tilde{\mathbf{G}} \mathbf{B}^{\mathrm{T}}, \mathbf{K}=\mathbf{B C}$. The equation $\mathbf{B C}=\mathbf{K}$ is

$$
b_{i k} c_{k j}=k_{i j} \text {. }
$$

But $\mathbf{K}$ is tridiagonal, so that $k_{i j}=0$, for $i=1,2, \ldots, n-2 ; j=i+2, \ldots, n$. The matrix $\mathbf{B}$ is lower bidiagonal, so that (5.9) gives

$$
b_{i, i-1} c_{i-1, j}+b_{i i} c_{i j}=0 \quad i=1,2, \ldots, n-1 ; j=i+2, \ldots, n .
$$

Thus

$$
b_{11} c_{1 j}=0 \quad j=3,4, \ldots, n
$$

and hence

$$
c_{i j}=0 \quad i=1,2, \ldots, n-2 ; j=i+2, \ldots, n .
$$

Now consider $\mathbf{C}=\tilde{\mathbf{G}} \mathbf{B}^{\mathrm{T}}$, which we write as

$$
c_{i j}=\tilde{g}_{i k} b_{j k}=\tilde{g}_{i, j-1} b_{j, j-1}+\tilde{g}_{i j} b_{j j} .
$$

When combined with (5.9), this gives

$$
\tilde{g}_{i, j-1} b_{j, j-1}+\tilde{g}_{i j} b_{j j}=0 \quad i=1,2, \ldots, n-2 ; j=i+2, \ldots, n .
$$

Since $\tilde{g}_{i j}=(-)^{i+j} g_{i j}$ and $\mathbf{G}$ is symmetric, we may write these equations as

$$
b_{j, j-1}\left[\begin{array}{c}
g_{j-1,1} \\
g_{j-1,2} \\
\vdots \\
g_{j-1, j-2}
\end{array}\right]=b_{j j}\left[\begin{array}{c}
g_{j 1} \\
g_{j 2} \\
\vdots \\
g_{j, j-2}
\end{array}\right] \quad j=3,4, \ldots, n .
$$

We will show that these equations mean that the compound matrix $\mathbf{G}_{2}$ of $2 \times 2$ minors of $\mathbf{G}$ has a pattern of zeros like that shown in figure 1. Starting from its left-hand end, the last row of $\mathbf{G}_{2}$ contains the terms

$$
G(n-1, n ; 1,2), G(n-1, n ; 1,3), \ldots, G(n-1, n ; 1, n-2), \ldots .
$$

By taking $j=n$ in (5.11), we see that all these minors are zero. The penultimate row of $\mathbf{G}_{2}$ starts with the terms

$$
G(n-2, n ; 1,2), \ldots, G(n-2, n ; 1, n-3) .
$$

We will show that these are all zero. To do so, we take the zero determinant

$$
\left|\begin{array}{ccc}
g_{n-2,1} & g_{n-2,1} & g_{n-2, k} \\
g_{n-1,1} & g_{n-1,1} & g_{n-1, k} \\
g_{n, 1} & g_{n, 1} & g_{n, k}
\end{array}\right|
$$

and expand it along its first column to give

$g_{n-2,1} G(n-1, n ; 1, k)-g_{n-1,1} G(n-2, n ; 1, k)+g_{n, 1} G(n-2, n-1 ; 1, k)=0$.

However, as we showed earlier, G is a positive matrix, so that $g_{n-2,1}, g_{n-1,1}$ and $g_{n, 1}$ are all positive. This means that if two of the minors in this equation are zero, then so is the third. But if $k=2,3, \ldots, n-3$, then (5.11) with $j=n-1$ shows that $G(n-2, n-1 ; 1, k)=0$, while (5.11) with $j=n$ shows that $G(n-1, n ; 1, k)=0$. Therefore, $G(n-2, n ; 1, k)=0, k=2,3, \ldots, n-3$. 
The row of $\mathbf{G}_{2}$, third from the bottom, starts with the terms $G(n-2, n-1 ; 1, k)$; these again are zero for $k=2,3, \ldots, n-3$. Proceeding in this way we find that $G(i, j ; 1, k)=0$ for $3 \leqslant i<j, k=2, \ldots, i-1$. This provides a non-increasing pattern of zeros for the columns of $\mathbf{G}_{2}$ in the lower triangle, and this will thus be transmitted to the corresponding compound matrix $\mathbf{G}_{2}^{\prime}$ of $\mathbf{G}^{\prime}$.

The matrix $\mathbf{G}^{\prime}$, like $\mathbf{G}$, is a positive matrix. To see this, we note that $g_{n 1}>0$, and hence, by (5.7), $g_{n 1}^{\prime}>0$. All the $2 \times 2$ minors of $\mathbf{G}^{\prime}$ are non-negative, because $\mathbf{G}^{\prime}$ is oscillatory; therefore,

$$
\mathbf{G}^{\prime}(i, n ; 1, i) \equiv g_{i 1}^{\prime} g_{n i}^{\prime}-g_{i i}^{\prime} g_{n 1}^{\prime} \geqslant 0 .
$$

However, $\mathbf{G}^{\prime}$ is positive definite so that $g_{i i}^{\prime}>0$ and hence $g_{i 1}^{\prime} g_{n i}^{\prime} \geqslant g_{i i}^{\prime} g_{n 1}^{\prime}>0$, so that $g_{i 1}^{\prime}>0$, i.e. the first column and last row of $\mathbf{G}^{\prime}$ are positive. Now

$$
G^{\prime}(i, n ; j, i)=g_{i j}^{\prime} g_{n i}^{\prime}-g_{i i}^{\prime} g_{n j}^{\prime} \geqslant 0
$$

so that $g_{i j}^{\prime} g_{n i}^{\prime} \geqslant g_{i i}^{\prime} g_{n j}^{\prime}>0$ and $g_{i j}^{\prime}>0$.

To construct $\mathbf{B}^{\prime}$ and $\mathbf{K}^{\prime}$ we must retrace the steps from (5.9) to (5.11) with the new matrix $\mathbf{G}^{\prime}$.

\section{Conclusions}

In the preceding sections, we have shown how we can construct families of isospectral systems $(\mathbf{K}, \mathbf{M})$, from one system. The widest family is that discussed in section 5. From one pair we construct the oscillatory matrix $\mathbf{G}$ and from that we may construct an infinity of other oscillatory matrices $\mathbf{G}^{\prime}$ by repeated shifts, with different shifts $\mu$. From the new $\mathbf{G}^{\prime}$ we construct pairs $\mathbf{K}^{\prime}$ and $\mathbf{M}^{\prime}$. It is still an open question whether, starting from one pair of $(\mathbf{K}, \mathbf{M})$ with eigenvalues $\left(\lambda_{i}\right)_{1}^{n}$ with $0<\lambda_{1}<\cdots<\lambda_{n}$, we may construct the complete family of pairs.

This paper is an extension of Gladwell (1995). There we considered a spring mass system for which $\mathbf{K}$ has the form (2.1), but $\mathbf{M}$ is diagonal, i.e. $\mathbf{M}=\operatorname{diag}\left(m_{1}, m_{2}, \ldots, m_{n}\right)$. For such a system $\mathbf{G}=\tilde{\mathbf{M}}^{-1 / 2} \tilde{\mathbf{K}} \tilde{\mathbf{M}}^{-1 / 2}$ is a Jacobi matrix. In that paper we proved that, from any Jacobi matrix $\mathbf{G}$, we could proceed by $n-1$ shifts $\mu_{1}, \ldots, \mu_{n-1}$, to any other isospectral Jacobi matrix G*, and we gave an explicit procedure for finding the appropriate shifts. We have not yet been able to generalize this result to two arbitrary isospectral oscillatory matrices $\mathbf{G}=\mathbf{B}^{-1} \tilde{\mathbf{K}} \mathbf{B}^{-\mathrm{T}}, \mathbf{G}^{*}=\mathbf{B}^{*-1} \tilde{\mathbf{K}}^{*} \mathbf{B}^{*-\mathrm{T}}$ constructed from pairs of Jacobi matrices $\tilde{\mathbf{K}}=\mathbf{A A}^{\mathrm{T}}, \mathbf{M}=\mathbf{B B}^{\mathrm{T}}, \tilde{\mathbf{K}}^{*}=\mathbf{A}^{*} \mathbf{A}^{* \mathrm{~T}}, \mathbf{M}^{*}=\mathbf{B}^{*} \mathbf{B}^{* \mathrm{~T}}$.

In this paper we have shown how to construct a family of systems which are isospectral to a given system. However, it still remains an open question of how to construct one pair of Jacobi matrices $\tilde{\mathbf{K}}$ and $\mathbf{M}$ such that (3.1) has given eigenvalues $\lambda_{1}, \lambda_{2}, \ldots, \lambda_{n}$ satisfying $0<\lambda_{1}<\lambda_{2}<\cdots<\lambda_{n}$. As an attempt to answer this question we present the following result.

Take $\mathbf{K}$ and $\mathbf{M}$ to be almost diagonal. If $k_{i i}=a_{i}$, where $0<a_{1}<a_{2}<\cdots<a_{n}$, $k_{i i}=-\varepsilon, m_{i i}=1, m_{i, i-1}=\varepsilon$, then, for small $\varepsilon$, the eigenvalues of $(\mathbf{K}, \mathbf{M})$ will be given by

$$
\lambda_{i}=a_{i}+\mathrm{o}\left(\varepsilon^{2}\right)
$$

We may extend this result to find a pair $\mathbf{K}$ and $\mathbf{M}$ with eigenvalues which difer from $\lambda_{i}$ by terms of order $\varepsilon^{4}$. We take

$$
\begin{aligned}
& k_{i i}=\lambda_{i} \quad m_{11}=1-d_{1} \varepsilon^{2} \quad m_{n n}=1+d_{n} \varepsilon^{2} \\
& m_{i i}=1 \quad i=2, \ldots, n-1 \quad-k_{i, i-1}=b_{i} \varepsilon=m_{i, i-1} \quad i=2, \ldots, n \text {. }
\end{aligned}
$$


We can easily show that if we choose

$$
\frac{d_{1} \lambda_{1}}{\left(1+\lambda_{1}\right)^{2}}=\frac{b_{1}^{2}}{\lambda_{2}-\lambda_{1}}=\frac{b_{2}^{2}}{\lambda_{3}-\lambda_{2}}=\cdots=\frac{b_{n-1}^{2}}{\lambda_{n}-\lambda_{n-1}}=\frac{d_{n} \lambda_{n}}{\left(1+\lambda_{n}\right)^{2}}
$$

then the eigenvalues of $\mathbf{K}$ and $\mathbf{M}$ are $\lambda_{i}+o\left(\varepsilon^{4}\right) ; \varepsilon$ must be kept small compared to the minimum spacing between eigenvalues. This result is unsatisfactory for the following reason. If we factorize $\mathbf{M}=\mathbf{B B}^{\mathrm{T}}$, then the elements of $\mathbf{B}^{-1}$ will decrease like $\varepsilon^{r}$, where $r$ is proportional to the distance away from the diagonal. Thus, even though $\mathbf{G}$ is actually a positive matrix, it will be practically banded, and so therefore will any other $\mathbf{G}^{\prime}$ derived from $\mathbf{G}$ by shifted QR.

We must not take both $\mathbf{K}$ and $\mathbf{M}$ to be almost diagonal. We may take $\mathbf{K}$ to be nearly diagonal, and $\mathbf{M}$ not, and form $\tilde{\mathbf{G}}=\mathbf{B}^{-1} \mathbf{K} \mathbf{B}^{-\mathrm{T}}$. Alternatively, we may take $\mathbf{M}$ to be almost diagonal, and $\mathbf{K}=\mathbf{A} \mathbf{A}^{\mathrm{T}}$ not, and form the oscillatory matrix $\mathbf{H}=\mathbf{A}^{-1} \mathbf{M} \mathbf{A}^{-\mathrm{T}}$ with eigenvalues $\lambda_{i}^{-1}$. This is a problem for further analytical or numerical research.

In this paper we assumed that the oscillatory matrix $\mathbf{G}$ was formed from reducing the generalized eigenvalue problem (3.1) to standard symmetric form. We assumed that the vibrating system was rod-like, so that both $\mathbf{K}$ and $\mathbf{M}$ were tridiagonal. If the system is beam-like, then both $\mathbf{K}$ and $\mathbf{M}$ will be pentadiagonal and, provided that they are formed from splines, as in Gladwell (1991), $\tilde{\mathbf{K}}$ and $\mathbf{M}$ will still be oscillatory so that the matrix $\mathbf{G}$ obtained after reduction to stardard symmetric form will also be oscillatory. This means that the conclusions we have drawn for the rod problem may be generalized to the beam problem.

Practical examples of isospectral finite-element rods and beams will be considered in a subsequent paper.

\section{References}

Boley D A and Golub G H 1987 A survey of matrix inverse eigenvalue problems Inverse Problems 3 595-622 Chu M T 1995 Inverse eigenvalue problems Preprint

Friswell M I and Mottershead J E 1995 Finite Element Model Updating in Structural Dynamics (Dordrecht: Kluwer) Gantmakher F P and Krein M G 1950 Oscillation Matrices and Kernels and Small Vibration of Mechanical Systems (Moscow, Leningrad: State Publishing House for Technical-Theoretical Literature) (1961 translation (Washington, DC: US Atomic Energy Commission))

Gladwell G M L 1986a Inverse Problems in Vibration (Dordrecht: Martinus Nijhoff)

Gladwell G M L 1986b Inverse problems in vibration Appl. Mech. Rev. 39 1013-18

Gladwell G M L 1991 Qualitative properties of finite element models III: spline models Int. Conf. Mechanics of Solids and Structures (Singapore)

Gladwell G M L 1995 On isospectral spring-mass systems Inverse Problems 11 591-602

Gladwell G M L 1996a Inverse problems in vibration Appl. Mech. Rev. 49 525-34

Gladwell G M L 1996b Total positivity and the QR algorithm Linear Alg. Appl. submitted

Gladwell G M L and Ahmadian H 1995 Generic element matrices suitable for finite element model updating Mech. Syst. Signal Proc. 9 601-14

Gordis J H 1996 On the analytic disassembly of structural matrices Modal Anal. 11 39-48

Karlin S 1968 Total Positivity (Stanford, CA: Stanford University Press)

Mottershead J E and Friswell M I 1993 Model updating in structural dynamics J. Sound Vib. 167 347-75 